\title{
Parkinsonism-hyperthermia syndrome and deep brain stimulation
}

\author{
Stanley N. Caroff, MD
}

Received: 8 January 2017/Accepted: 31 January 2017/Published online: 14 February 2017

(c) Canadian Anesthesiologists' Society 2017

\section{To the Editor,}

In their comprehensive review, Yeoh et al. provide a scholarly summary of the challenges posed by deep brain stimulation (DBS) devices while providing effective, safe anesthesia for a diversity of surgical and imaging procedures. ${ }^{1}$ It is a timely, useful clinical review, especially given the increasing prevalence of patients with implanted DBS devices. As the authors recommend turning off DBS devices during procedures to prevent harmful interactions and consequences, it may be worthwhile to remind clinicians of the potential risk of parkinsonism-hyperthermia syndrome (PHS) as a complication in patients with Parkinson's disease.

Parkinsonism-hyperthermia syndrome has been well described in patients with Parkinson's disease after oral dopaminergic therapy is reduced, discontinued, or becomes ineffective. $^{2}$ Regardless of the reason for treatment withdrawal, patients may develop a potentially fatal central nervous system hypo-dopaminergic crisis within hours to days, characterized by hyperthermia, muscle rigidity, autonomic instability, and altered consciousness. ${ }^{2}$ Complications of PHS include acute renal failure, aspiration pneumonia, deep venous thrombosis leading to pulmonary embolism, persistent neurologic sequelae, and disseminated intravascular coagulation. PHS is indistinguishable from - and should be considered in the

This letter is accompanied by a reply. Please see Can J Anesth 2017; 64: this issue

S. N. Caroff, MD $(\bowtie)$

Perelman School of Medicine at the University of Pennsylvania,

Philadelphia, PA, USA differential diagnosis of - other drug-related hyperthermic disorders (e.g., neuroleptic malignant syndrome, serotonin syndrome).

Parkinsonism-hyperthermia syndrome has been reported in the perioperative setting when oral dopaminergic therapy is withheld prior to surgery or not restarted quickly enough afterward. ${ }^{3}$ In this setting, PHS may be mistaken for anesthetic-induced malignant hyperthermia $(\mathrm{MH}) .^{3}$ In contrast to the use of dantrolene in $\mathrm{MH}$, however, treatment of PHS consists principally of rapid reinstitution of antiparkinsonian therapy in addition to supportive care.

Parkinsonism-hyperthermia syndrome has occurred when oral therapy was reduced too rapidly during the transition period after implantation of DBS devices. ${ }^{4}$ Recently, PHS has also been reported following discontinuation or failure of DBS devices or batteries in patients with Parkinson's disease, independent of changes in dopaminergic medications. ${ }^{5}$ Artusi et al. reviewed five such cases (three of which were fatal) that occurred within one to six days after cessation of stimulation. ${ }^{5}$

The recommendation by Yeoh et al. to turn off DBS devices temporarily during procedures, thereby preventing serious interactions, appears well founded based on the evidence. Their corollary advice to consult with DBS specialists prior to and following anesthesia and to restart DBS devices before reversal of anesthesia appears prudent as well, with the additional caveat to be aware of the signs of PHS, its resemblance to $\mathrm{MH}$, and the need to act expeditiously to reverse its symptoms.

Conflict of interest disclosures Dr. Caroff has received research grant support from Sunovion Pharmaceuticals and has served as a consultant to Neurocrine Biosciences, Teva Pharmaceuticals, and Eagle Pharmaceuticals. 
Editorial responsibility This submission was handled by Dr. Hilary P. Grocott, Editor-in-Chief, Canadian Journal of Anesthesia.

Funding No funding was received for this letter.

\section{References}

1. Yeoh TY, Manninen P, Kalia SK, Venkatraghavan L. Anesthesia considerations for patients with an implanted deep brain stimulator undergoing surgery: a review and update. Can J Anesth 2016; DOI:10.1007/s12630-016-0794-8.
2. Newman EJ, Grosset DG, Kennedy PG. The parkinsonismhyperpyrexia syndrome. Neurocrit Care 2009; 10: 136-40.

3. Stotz M, Thummler D, Schurch M, Renggli JC, Urwyler A, Pargger $H$. Fulminant neuroleptic malignant syndrome after perioperative withdrawal of antiParkinsonian medication. Br J Anaesth 2004; 93: 868-71.

4. Factor SA. Fatal Parkinsonism-hyperpyrexia syndrome in a Parkinson's disease patient while actively treated with deep brain stimulation. Mov Disord 2007; 22: 148-9.

5. Artusi CA, Merola A, Espay AJ, et al. Parkinsonism-hyperpyrexia syndrome and deep brain stimulation. J Neurol 2015; 262: 2780-2. 